\title{
Reassessment of the taxonomic position of Vibrio iliopiscarius (Onarheim et al. 1994) and proposal for Photobacterium iliopiscarium comb. nov.
}

\author{
Hidetoshi Urakawa, Kumiko Kita-Tsukamoto and Kouichi Ohwada
}

\begin{abstract}
Author for correspondence: Kumiko Kita-Tsukamoto. Tel: +8135351 6484. Fax: +81353516482. e-mail: tukamoto@ori.u-tokyo.ac.jp
\end{abstract}

Ocean Research Institute, University of Tokyo, 1-15-1 Minamidai, Nakano-ku, Tokyo 164-8639, Japan

\begin{abstract}
The phylogenetic position of Vibrio iliopiscarius was inferred by the maximumlikelihood, maximum-parsimony and neighbour-joining methods on the basis of almost complete 16S rRNA gene sequences. The results showed that this species falls into the same cluster as Photobacterium species and is clearly distinct from other Vibrio species. Its nearest phylogenetic neighbour is Photobacterium phosphoreum. From these results, it is concluded that $V$. iliopiscarius should be reclassified as Photobacterium iliopiscarium comb. nov., the type strain of which is PS1' (= ATCC 51760').
\end{abstract}

Keywords: Vibrio iliopiscarius, Photobacterium iliopiscarium, Vibrionaceae, Vibrio, Photobacterium

\section{INTRODUCTION}

Vibrio iliopiscarius was isolated from the intestines of fish (herring, coal fish, cod and salmon) living in cold seawater and reported as a new species of the genus Vibrio by Onarheim et al. (1994). These authors determined the phenotypic characteristics and partial 16S rRNA sequence of $V$. iliopiscarius and concluded that this species was different from all the described species of the genus Vibrio, its nearest phylogenetic neighbours being Vibrio salmonicida and Vibrio fischeri (Onarheim et al., 1994). The type strain of $V$. iliopiscarius is ATCC $51760^{\mathrm{T}}$.

Recently, RFLP analysis on the basis of PCRamplified 16S rRNA genes among the members of the family Vibrionaceae was performed (Urakawa et al., 1997, 1998). The RFLP profile of $V$. iliopiscarius (ATCC $51760^{\mathrm{T}}$ ) by $H h a \mathrm{I}$ digestion showed the same restriction fragment pattern as did species of the genus Photobacterium (Urakawa et al., 1998). In addition, this species showed the same RFLP profile as did Photobacterium phosphoreum and Photobacterium leiognathi with the four-base-specific restriction enzymes HhaI, DdeI, RsaI, Sau3AI and MspI (Urakawa et al.,

Abbreviations: ML, maximum likelihood; MP, maximum parsimony; NJ, neighbour-joining.

The DDBJ accession number for the 165 rRNA gene sequence of $P$. iliopiscarium strain $\mathrm{PS}^{\top}$ is $\mathrm{AB} 000278$.
1997). Considering these results, the taxonomic position of $V$. iliopiscarius was reassessed on the basis of almost complete $16 \mathrm{~S}$ rRNA gene sequence data.

\section{METHODS}

DNA extraction and PCR amplification of 16S rRNA genes were carried out as described previously (Urakawa et al., 1997). The PCR product was purified and concentrated with a Microcon-100 micro-concentrator (Amicon) according to the manufacturer's instructions. Sequencing was carried out with a SequiTherm long-read cycle sequencing kit (Epicentre Technologies) and an automated sequencer (ALF DNA Sequencer; Amersham-Pharmacia Biotech). Sequences were aligned by using the CLUSTAL w program (version 1.60) (Thompson et al., 1994) and then realigned manually with the MACCLADE 3.06 software (Maddison \& Maddison, 1992). Nucleotide positions of ambiguous alignment and gaps were omitted from subsequent phylogenetic analysis. Maximumlikelihood (ML) analysis was performed by using the NUCML program in the MOLPHY package (version 2.3) (Adachi \& Hasegawa, 1996). First, the neighbour-joining (NJ) tree (Saitou \& Nei, 1987) was inferred by using a distance matrix estimated by the ML method. The optimal transition/ transversion ratio was calculated by the HKY85 model (Hasegawa et al., 1985). The tree thus obtained was analysed further by the local rearrangement method to search the ML tree. Bootstrap probability was estimated by the resampling of estimated log-likelihood method (Kishino et al., 1990). Maximum-parsimony (MP) analysis was performed by using the PAUP program (version 3.1.1) (Swofford, 1993) with a heuristic search. Bootstrap confidence values were obtained with 100 resamplings with an option of stepwise addition. 
NJ analysis was also performed by using the CLUSTAL w program. The NJ tree was constructed from the distance matrix calculated by the algorithm of Kimura's twoparameter model (Kimura, 1980). Bootstrap confidence values were obtained with 1000 resamplings.

\section{RESULTS AND DISCUSSION}

Three different phylogenetic analyses were carried out with sequence data from almost all Vibrio species available in the databases (DDBJ, EMBL and GenBank). A total of 1244 aligned sites were used for the analysis. The ML tree is shown in Fig. 1. $V$. iliopiscarius clustered with the genus Photobacterium with high bootstrap confidence values and was clearly distinguished from other Vibrio species (Fig. 1). The closest relative to $V$. iliopiscarius was $P$. phosphoreum (99.7\% identity). This relationship was also supported by both the MP and NJ methods with $100 \%$ bootstrap confidence values (data not shown). The data show clearly that $V$. iliopiscarius should be transferred from the genus Vibrio to the genus Photobacterium as Photobacterium iliopiscarium comb. nov. In addition to the phylogenetic information, several phenotypic traits of $V$.iliopiscarius that are common to most or all Photobacterium species and rarely seen in Vibrio species support the transfer to the genus Photobacterium, such as gas production from glucose, positive Voges-Proskauer, arginine dihydrolase and lysine decarboxylase tests and the inability to use

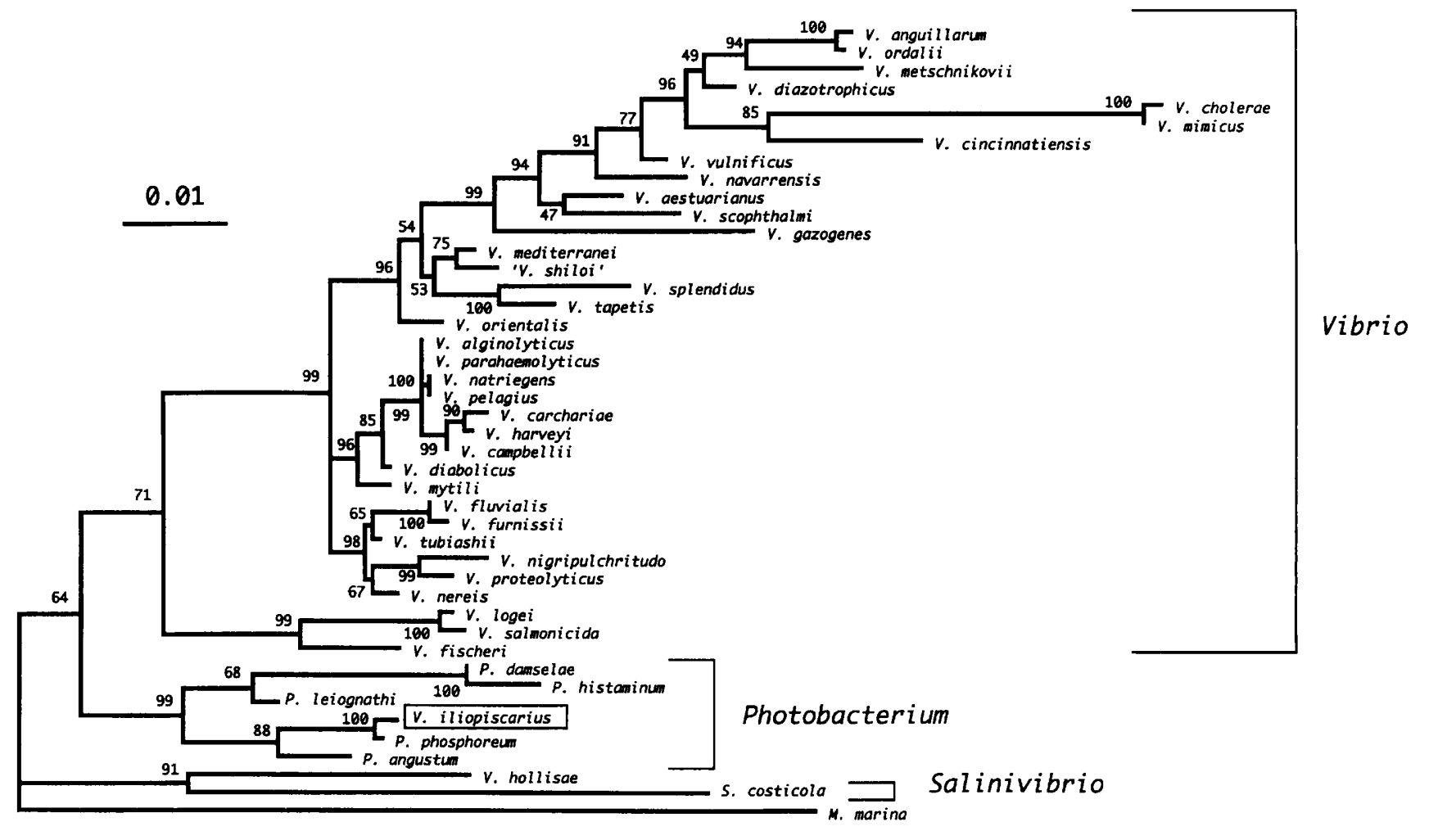

Fig. 1. Maximum-likelihood tree deduced from complete $16 \mathrm{~S}$ rRNA sequences of $V$. iliopiscarius and members of the genera Vibrio, Photobacterium, Salinivibrio and Moritella (HKY85 model; alpha/beta $=2 \cdot 168 ; \ln L=-6188 \cdot 20$ ). Bar, one nucleotide substitution per 100 nucleotides. Bootstrap confidence values (percentage) obtained with 1000 resamplings are given at the branch-points. Accession numbers of reference organisms used in the phylogenetic analysis are: Moritella marina (=Vibrio marinus) NCIMB 1144, X82142; Photobacterium angustum ATCC 25915 ${ }^{\top}$, D25307; Photobacterium damselae ATCC 33539 ${ }^{\top}, X^{2} 74700$; Photobacterium histaminum C-8 ${ }^{\top}$, D25308; P. leiognathi ATCC $25521^{\top}$, D25309; P. phosphoreum ATCC 11040', D25310; Salinivibrio costicola NCIMB 701', X95527; Vibrio aestuarianus ATCC 35048' ${ }^{\top}$ X74689; Vibrio alginolyticus ATCC 17749 ${ }^{\top}, X 74690$; Vibrio anguillarum ATCC 19264 ${ }^{\top}, X 16895$; Vibrio campbellii ATCC $25920^{\top}$, X74692; Vibrio carchariae ATCC 35084 ${ }^{\top}, X^{2} 74693$; Vibrio cholerae ATCC 14033, X74694; Vibrio cincinnatiensis ATCC $35912^{\top}, \mathrm{X74698}$; Vibrio diabolicus HE800 ${ }^{\top}, \mathrm{X} 99762$; Vibrio diazotrophicus ATCC $33466^{\top}, \mathrm{X74701}^{\circ} \mathrm{V}$. fischeri ATCC $7744^{\top}, X 74702$; Vibrio fluvialis ATCC $33809^{\top},{ }^{2} \times 74703$; Vibrio furnissii ATCC $35016^{\top}, X 74704$; Vibrio

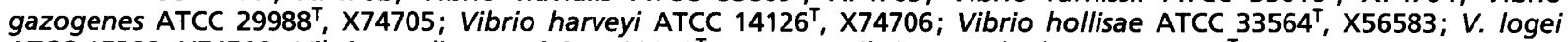
ATCC 15382, X74708; Vibrio mediterranei CIP 103203 ${ }^{\top}$, X74710; Vibrio metschnikovii CIP 69.14T' X74711; Vibrio mimicus ATCC $33653^{\top}$, X74713; Vibrio mytili CECT $632^{\top}$, X99761; Vibrio natriegens ATCC 14048 ${ }^{\top}$, X74714; Vibrio navarrensis CIP $103381^{\top}$, X74715; Vibrio nereis ATCC $25917^{\top}$, X74716; Vibrio nigripulchritudo ATCC $27043^{\top}, X^{\top} 7417$; Vibrio ordalii ATCC $33509^{\top}, X^{\top} 74718$; Vibrio orientalis ATCC $33934^{\top}$, X74719; Vibrio parahaemolyticus ATCC $17802^{\top}$, X74720; Vibrio pelagius ATCC 25916 ${ }^{\top}$, X74722; Vibrio proteolyticus ATCC 15338 ${ }^{\top}, X^{2} 74723 ; V$. salmonicida NCMB 2262 ${ }^{\top}$, X70643; Vibrio scophthalmi CECT 4638', U46579; 'Vibrio shiloi' AK-1, AF007115; Vibrio splendidus ATCC $33125^{\top}$, X74724; Vibrio tapetis CECT 4600', Y08430; Vibrio tubiashii ATCC 19109' ${ }^{\top}, \mathrm{X74725}^{\top}$; and Vibrio vulnificus ATCC $27562^{\top}, \mathrm{X}^{\top} \mathrm{X726.}$ 
(a)

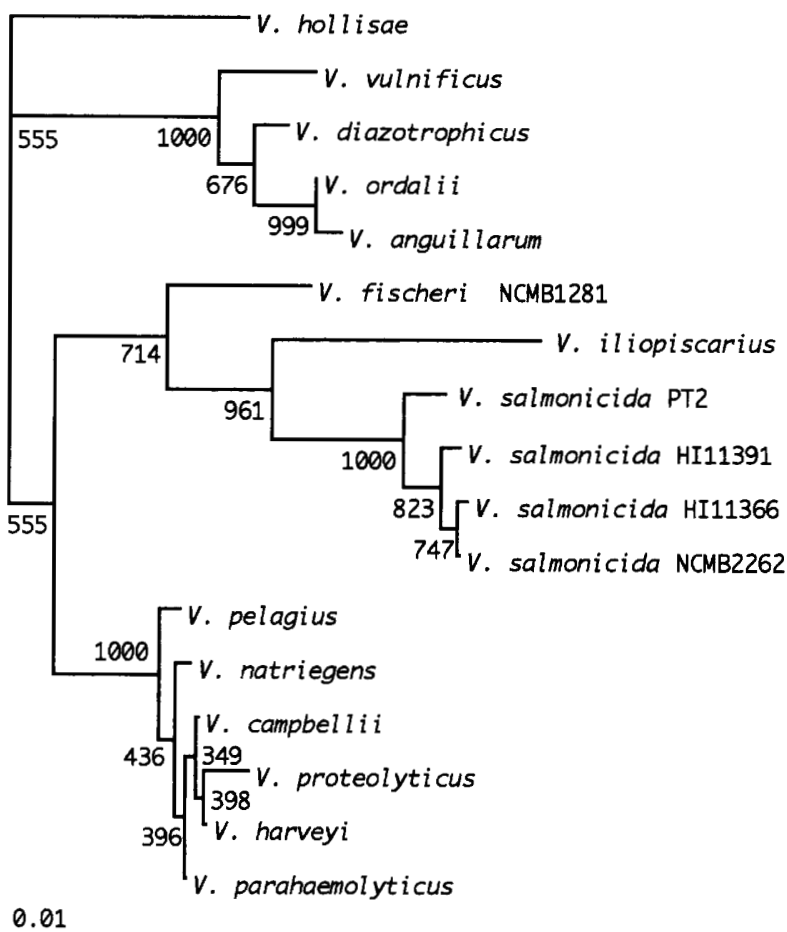

(b)

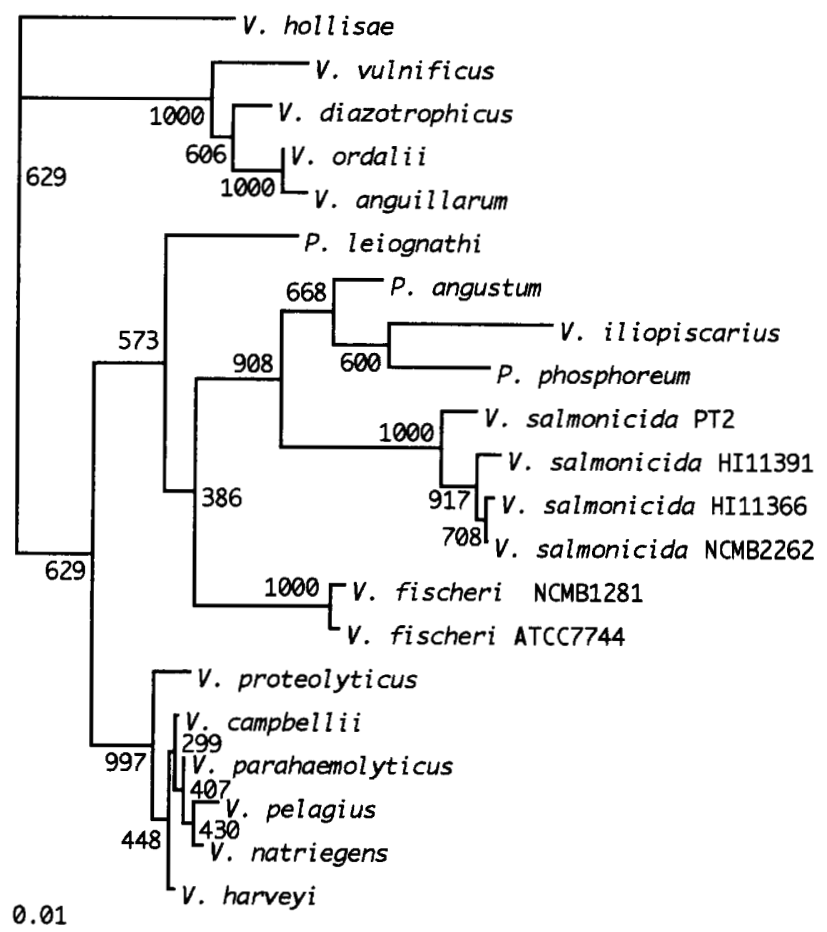

Fig. 2. Neighbour-joining trees reproduced from sequence data of Onarheim et al. (1994) (a) and rearranged data (b). The 550 aligned sites described by Onarheim et al. (1994) were used for phylogenetic analysis. Bar, one nucleotide substitution per 100 nucleotides. Bootstrap confidence values obtained with 1000 resamplings are given at the branchpoints. Accession numbers of reference organisms used in the phylogenetic analysis are as listed in the legend to Fig. 1 with the addition of: $V$. fischeri NCMB 1281, X70640; V. iliopiscarius PS1 ${ }^{\top}$, X70636; V. pelagius NCMB 1900, X70642; V. salmonicida HI 11366, X70638; V. salmonicida HI 11391, X70639; and V. salmonicida PT2, X70637.

mannitol and to hydrolyse gelatin (Onarheim et al., 1994). This phenotypic concordance reinforces our phylogenetic conclusion.

Stackebrandt \& Rainey (1995) have insisted that phylogenetic positions of taxa should be based on analysis of complete sequences. However, the phylogenetic position of $V$. iliopiscarius was determined on the basis of partial sequence data (Onarheim et al., 1994). These authors stated that the important factors are that the number of nucleotides compared is statistically meaningful, that for closely related organisms variable regions are included in the analysis and that the sequences compared are appropriately aligned. Onarheim et al. (1994) compared V. iliopiscarius only with Vibrio species and did not include Photobacterium species. Whether this misclassification was due to the use of partial sequence data or an inappropriate combination of species in the phylogenetic analysis is not clear. A phylogenetic tree has been reconstructed by using the same combination of species and sequence data used in this original analysis; the same topology among $V$. salmonicida, $V$. fischeri and $V$. iliopiscarius that was shown by Onarheim et al. (1994) was reproduced (Fig. 2a). In this tree, $V$. iliopiscarius clustered with $V$. salmonicida as shown by
Onarheim et al. (1994). 16S rRNA sequence data from Photobacterium species and another strain of $V$.fischeri were then added to the data used by Onarheim et al. (1994) to examine the validity of the combination of species used in their analysis (Fig. 2b). In this tree, $V$. salmonicida, $V$. fischeri, $V$. iliopiscarius and other Photobacterium species fell into the same cluster. Onarheim et al. (1994) used 550 nucleotides in their analysis, consisting of nucleotides $1-185,300-508$ and 932-1089. From these results, it is assumed that the partial sequences used by Onarheim et al. (1994) were not sufficient to separate Photobacterium species from $V$. salmonicida, $V$. fischeri and other Vibrio species. As shown in Fig. 1, phylogenetic analysis with complete sequences showed that $V$. iliopiscarius did not fall within the Vibrio cluster and that it was clearly separated from $V$. salmonicida, Vibrio logei and $V$. fischeri. Thus, it is clear that the misclassification was caused by the effect of partial sequence analysis.

In summary, the taxonomic position of $V$. iliopiscarius has been re-examined. The phylogenetic position of this species, on the basis of almost complete 16S rRNA sequences, was determined by ML, MP and NJ analyses. $V$. iliopiscarius was most closely related to $P$. phosphoreum in all phylogenetic analyses carried out in 
this study, with high bootstrap probabilities. From these results, $V$. iliopiscarius should be transferred to the genus Photobacterium, as P. iliopiscarium comb. nov.

\section{Emended description of Photobacterium iliopiscarium (Onarheim et al. 1994) Urakawa, Kita-Tsukamoto and Ohwada comb. nov.}

The description of Photobacterium iliopiscarium comb. nov. is identical to that given by Onarheim et al. (1994) except the description of 16S rRNA gene sequence analysis. The type strain is $\operatorname{PS}^{\mathrm{T}}\left(=\right.$ ATCC $\left.51760^{\mathrm{T}}\right)$.

\section{ACKNOWLEDGEMENTS}

This study was supported by Research Fellowships of the Japan Society for the Promotion of Science for Young Scientists.

\section{REFERENCES}

Adachi, J. \& Hasegawa, M. (1996). MOLPHY version 2.3. Programs for Molecular Phylogenetics Based on Maximum Likelihood (Computer Science Monographs no. 28). Tokyo: Institute of Statistical Mathematics.

Hasegawa, M., Kishino, H. \& Yano, T. (1985). Dating of the human-ape splitting by a molecular clock of mitochondrial DNA. J Mol Evol 22, 160-174.

Kimura, M. (1980). A simple method for estimating evolutionary rates of base substitutions through comparative studies of nucleotide sequences. $J$ Mol Evol 16, 111-120.
Kishino, H., Miyata, T. \& Hasegawa, M. (1990). Maximum likelihood inference of protein phylogeny, and the origin of chloroplasts. $J$ Mol Evol 31, 151-160.

Maddison, W. P. \& Maddison, D. R. (1992). MACCLADE, version 3.0. Sunderland, MA: Sinauer.

Onarheim, A. M., Wiik, R., Burghardt, J. \& Stackebrandt, E. (1994). Characterization and identification of two Vibrio species indigenous to the intestine of fish in cold sea water; description of Vibrio iliopiscarius sp. nov. Syst Appl Microbiol 17, 370-379.

Saitou, N. \& Nei, M. (1987). The neighbor-joining method: a new method for reconstructing phylogenetic trees. Mol Biol Evol 4, 406-425.

Stackebrandt, E. \& Rainey, F. A. (1995). Partial and complete $16 \mathrm{~S}$ rDNA sequences, their use in generation of $16 \mathrm{~S}$ rDNA phylogenetic trees and their implications in molecular ecological studies. In Molecular Microbial Ecology Manual, pp. 3.1.1/1-3.1.1/17. Edited by A. D. L. Akkermans, J. D. van Elsas \& F. J. de Bruijn. Dordrecht: Kluwer.

Swofford, D. L. (1993). PAUP: Phylogenetic Analysis Using Parsimony. Champaign, IL: Illinois Natural History Survey.

Thompson, J. D., Higgins, D. G. \& Gibson, T. J. (1994). CLUSTAL $\mathrm{W}$ : improving the sensitivity of progressive multiple sequence alignment through sequence weighting, position-specific gap penalties and weight matrix choice. Nucleic Acids Res 22, 4673-4680.

Urakawa, H., Kita-Tsukamoto, K. \& Ohwada, K. (1997). 16S rDNA genotyping using PCR/RFLP (restriction fragment length polymorphism) analysis among the family Vibrionaceae. FEMS Microbiol Lett 152, 125-132.

Urakawa, H., Kita-Tsukamoto, K. \& Ohwada, K. (1998). A new approach to separate the genus Photobacterium from Vibrio with RFLP patterns by $H$ hal digestion of PCR-amplified $16 \mathrm{~S}$ rDNA. Curr Microbiol 36, 171-174. 